\title{
Evaluation of Occupational Safety and Health Practices by LPG Cylinder Retailers in Kiambu County, Kenya.
}

\author{
James Karori Nyabuto ${ }^{1}$, Charles Mburu, PhD $^{1}$, Margaret Gichuhi, PhD ${ }^{1}$ \\ ${ }^{1}$ Institute of Energy and Environmental Technology, Jomo Kenyatta University of Agriculture and Technology, Kenya.
}

\author{
DOI: 10.29322/IJSRP.10.07.2020.p10368 \\ http://dx.doi.org/10.29322/IJSRP.10.07.2020.p10368
}

\begin{abstract}
The study aimed at examining the current OSH practices by Lpg cylinder retailers in Kiambu County. Stratified purposive sampling was employed; raw data collected through observation, use of interview schedules, and analyzed using SPSS ver.25. It was established that $71 \%$ of the respondents did not employ safe OSH practices in their operations. The association of cylinder handling and education level of the respondents was statistically significant at $95 \%$ confidence level with $X^{2}(d f=2)=14.193$, since $p=<.001$. It was ascertained that $29.2 \%$ and $46.7 \%$ of the $13 \mathrm{~kg}$ and $6 \mathrm{~kg}$ cylinders respectively were non-conforming regarding weight measurements and non-destructive testing. Ignorance and lack of training and sensitization on Lpg safety were key challenges hindering application of good OSH practices. The study recommends routine inspections and compliance monitoring, by EPRA, in all Lpg retail centers. Moreover, engineering breakthroughs should be sought after in Lpg cylinder safety advancement.
\end{abstract}

Index Terms- Lpg cylinder safety, OSH , Retailers, EPRA

\section{INTRODUCTION}

Liquefied petroleum gas $(\mathrm{Lpg})$ cylinder retailers face the risk of serious injury or death while undertaking the retailing activities. The risk may also spill over to consumers. There is, therefore, a need for providing simple, practical advice on eliminating /reducing the risks associated with retailing of Lpg in cylinders. For this to be effectuated, the situation on the ground must be well understood. This then necessitated a study on the current occupational safety and health (OSH) practices, and challenges Impeding the application of good OSH practices in this trade, with an overall aim of promoting a positive safety culture.

\section{MATERIALS AND METHODS}

\subsection{Study design}

The study employed descriptive and diagnostic research designs. Descriptive research studies are concerned with describing the characteristics of an individual or group: This was employed to capture and describe the various OSH practices by the lpg cylinder retailers at their workplaces. Diagnostic research studies determine the frequency with which something occurs or its association with something else. (Kothari, 2004). This was employed during the lpg cylinder measurements and non-destructive cylinder testing.

\subsection{Study area and population}

The study area was Kiambu County, one of the 47 counties in the Republic of Kenya. It is in the central region and covers a total area of 2,543.5 Km². (County Government of Kiambu, 2015). The county has various urban centres namely: Thika, Juja, Ruiru Gatundu, Kiambu, Kikuyu, Karuri, and Limuru towns. (Kiambu County annual development plan, 2017). The study population comprised of 400 Lpg cylinder retailers undertaking the Lpg cylinder retail business, sampled from the selected study sites during the data collection period. (November, 2018 to February, 2019).

\subsection{Sampling method}

Stratified purposive sampling was employed. Thika, Limuru, and Kiambu towns were purposively selected from the population strata. The study sites were arrived at after taking into consideration the budget, time, personnel, and other resource limitations.

\subsection{Sample size determination}

Using the population in 2.2 above, the sample size was determined using the Barlett et al. sample size determination table. With the data being categorical, a selected margin of error of 0.05 , a standard variate value of 1.96 at $95 \%$ confidence level and a recommended population proportion of 0.50; the sample size determination table gives the sample size to use for the given population of 200 retailers in Thika town, 100 retailers in Kiambu town and 100 retailers in Limuru town to be 132, 80 and 80 respectively. (Bartlett et al., 2001) 


\subsection{Research instruments}

The measurement tools designed to obtain data from the research subjects were observation and interview schedules. Measurements and non-destructive tests were also undertaken where applicable.

\subsection{Pilot testing}

A pre-test was carried out in Kenyatta Road, to measure the validity and reliability of the research instruments. The pre-test targeted a sample size of 5 respondents in which all of them responded to the research instruments.

\subsection{Data processing and analysis}

Raw data from the field was coded, classified, checked for errors, and summarised. Thereupon, the data was analyzed using SPSS version 25, and Microsoft excel version 2019. Results of data analysis were organized and presented in the form of tables, pie charts, and bar graphs.

\subsection{Ethical consideration}

Respondents in the study had detailed information about the aim and objectives of the study. Permission to carry out the study was first sought from the university, then respondents in the select study sites. Confidentiality of the respondents was protected in that no names or personal information was required in the applicable research instrument, and no coercion or undue influence was exercised.

III. RESULTS AND DISCUSSION 


\subsection{Current Occupational safety and health practices}

The observations were made under natural conditions and findings presented below. Details of the cylinder brand owners and respondents were withheld for the protection of the dignity of the subjects, respect for anonymity, and confidentiality.

A fire safety risk assessment has been undertaken in the workplace

There is adequate lighting in the cylinder storage area

Workplace Inspections is undertaken regularly.

Material/product data sheets are available at the workplace

The Storage area is properly constructed

There's segregation of empty cylinders from full cylinders

Cylinders are well maintained with appropriate labelling

Cylinders are transported in a safe manner

Cylinder handling is done in a safe manner.

Cylinders are stored in a safe/proper manner

Housekeeping is properly done

\section{$3.60 \%$}

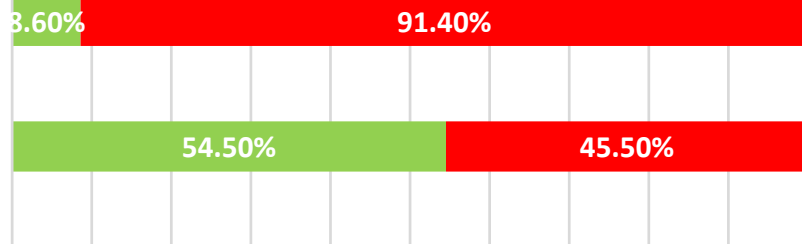

$12.30 \%$

$87.70 \%$
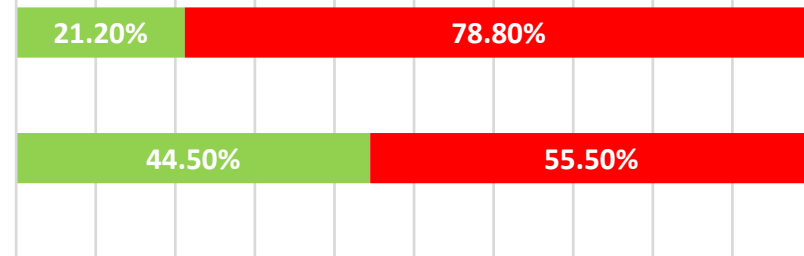

\section{$17.80 \%$}

$82.20 \%$
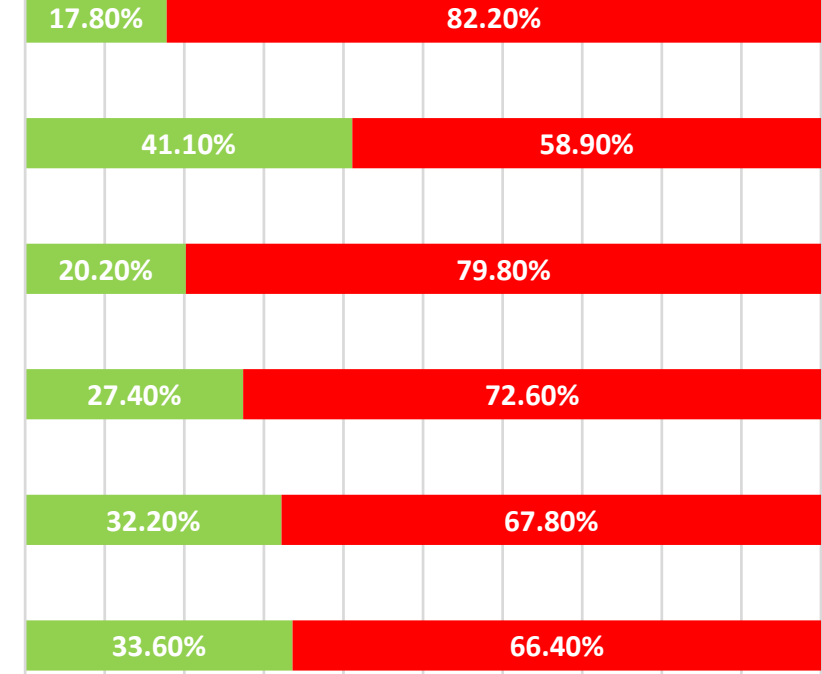

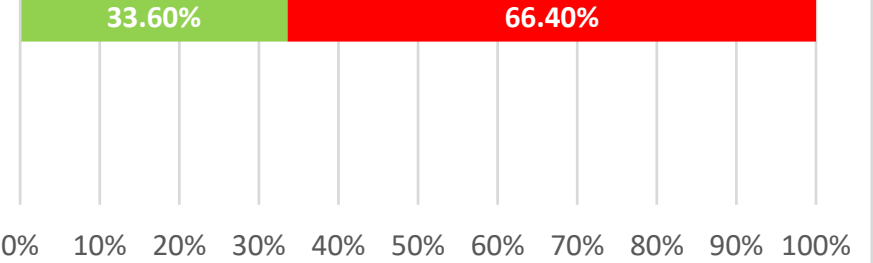

Agree $\square$ Disagree

Figure 1: Current occupational safety and health practices in LPG cylinder retail business 
Table 1: Association between respondents' demographics and housekeeping.

\begin{tabular}{|c|c|c|c|c|}
\hline \multirow[t]{2}{*}{ Variable } & \multirow[t]{2}{*}{ Category } & \multicolumn{3}{|c|}{ Housekeeping properly done. } \\
\hline & & No & Yes & Chi-Square \\
\hline \multirow[t]{2}{*}{ Gender } & Male & $69 \%$ & $31 \%$ & $\mathrm{X}^{2}=3.816, \mathrm{df}=1, \mathrm{p}=.051$ \\
\hline & Female & $55 \%$ & $45 \%$ & \\
\hline \multirow[t]{3}{*}{ Age } & $18-30$ years & $68 \%$ & $32 \%$ & \\
\hline & $31-40$ years & $71 \%$ & $29 \%$ & $\mathrm{X}^{2}=7.268, \mathrm{df}=2, \mathrm{p}=.026$ \\
\hline & $>40$ years & $49 \%$ & $51 \%$ & \\
\hline \multirow[t]{3}{*}{ Education level } & primary & $66 \%$ & $34 \%$ & \\
\hline & Secondary & $63 \%$ & $37 \%$ & $\mathrm{X}^{2}=3.479, \mathrm{df}=2, \mathrm{p}=.176$ \\
\hline & Tertiary & $75 \%$ & $25 \%$ & \\
\hline \multirow[t]{3}{*}{$\begin{array}{l}\text { Lpg retailing } \\
\text { experience }\end{array}$} & $<5$ years & $74 \%$ & $26 \%$ & \\
\hline & $6-10$ years & $59 \%$ & $41 \%$ & $X^{2}=17.303, d f=2, p=<.001$ \\
\hline & $>10$ years & $66 \%$ & $34 \%$ & \\
\hline
\end{tabular}

Table 2: Association between respondents' demographics and cylinder storage.

\begin{tabular}{lllll}
\hline Variable & Category & \multicolumn{3}{c}{ Cylinder storage in a safe manner } \\
& & No & Yes & Chi-Square \\
\cline { 2 - 4 } Gender & Male & $67 \%$ & $33 \%$ & $\mathrm{X}^{2}=0.107, \mathrm{df}=1, \mathrm{p}=.744$ \\
& Female & $70 \%$ & $30 \%$ & \\
& & & & \\
Age & $18-30$ years & $64 \%$ & $36 \%$ & \\
& $31-40$ years & $78 \%$ & $22 \%$ & $\mathrm{X}^{2}=16.755, \mathrm{df}=2, \mathrm{p}=<.001$ \\
& $>40$ years & $47 \%$ & $53 \%$ & \\
\multirow{3}{*}{ Education level } & & & & \\
& primary & $66 \%$ & $34 \%$ & \\
& Secondary & $66 \%$ & $34 \%$ & $\mathrm{X}^{2}=1.543, \mathrm{df}=2, \mathrm{p}=.462$ \\
& Tertiary & $74 \%$ & $26 \%$ & \\
& & & & \\
Lpg retailing experience & $<5$ years & $79 \%$ & $21 \%$ & \\
& & & & \\
& $6-10$ years & $51 \%$ & $49 \%$ & $\mathrm{X}^{2}=27.110, \mathrm{df}=2, \mathrm{p}=<.001$ \\
& $>10$ years & $48 \%$ & $52 \%$ & \\
\hline
\end{tabular}


Table 3: Association between respondents' demographics and cylinder handling.

\begin{tabular}{|c|c|c|c|c|}
\hline \multirow[t]{2}{*}{ Variable } & \multirow[t]{2}{*}{ Category } & \multicolumn{3}{|c|}{ Cylinder handling in a safe manner } \\
\hline & & No & Yes & Chi-Square \\
\hline \multirow[t]{2}{*}{ Gender } & Male & $69 \%$ & $31 \%$ & $\mathrm{X}^{2}=7.731, \mathrm{df}=1, \mathrm{p}=.005$ \\
\hline & Female & $88 \%$ & $12 \%$ & \\
\hline \multirow[t]{3}{*}{ Age } & $18-30$ years & $59 \%$ & $41 \%$ & \\
\hline & $31-40$ years & $81 \%$ & $19 \%$ & $X^{2}=17.471, d f=2, p=<.001$ \\
\hline & $>40$ years & $84 \%$ & $16 \%$ & \\
\hline \multirow[t]{3}{*}{ Education level } & primary & $58 \%$ & $42 \%$ & \\
\hline & Secondary & $74 \%$ & $26 \%$ & $\mathrm{X}^{2}=14.193, \mathrm{df}=2, \mathrm{p}=.001$ \\
\hline & Tertiary & $86 \%$ & $14 \%$ & \\
\hline \multirow[t]{3}{*}{ Lpg retailing experience } & $<5$ years & $81 \%$ & $19 \%$ & \\
\hline & $6-10$ years & $56 \%$ & $44 \%$ & $\mathrm{X}^{2}=19.459, \mathrm{df}=2, \mathrm{p}=<.001$ \\
\hline & $>10$ years & $76 \%$ & $24 \%$ & \\
\hline
\end{tabular}

Table 4: Association between respondents' demographics and cylinder transportation.

\begin{tabular}{|c|c|c|c|c|}
\hline \multirow{2}{*}{ Variable } & \multirow{2}{*}{ Category } & \multicolumn{3}{|c|}{ Cylinders transported in a safe manner } \\
\hline & & No & Yes & Chi-Square \\
\hline \multirow[t]{2}{*}{ Gender } & Male & $77 \%$ & $23 \%$ & $\mathrm{X}^{2}=5.465, \mathrm{df}=1, \mathrm{p}=.019$ \\
\hline & Female & $91 \%$ & $9 \%$ & \\
\hline \multirow[t]{3}{*}{ Age } & $18-30$ years & $79 \%$ & $21 \%$ & \\
\hline & $31-40$ years & $80 \%$ & $20 \%$ & $\mathrm{X}^{2}=0.100, \mathrm{df}=2, \mathrm{p}=.951$ \\
\hline & $>40$ years & $81 \%$ & $19 \%$ & \\
\hline \multirow[t]{3}{*}{ Education level } & primary & $80 \%$ & $20 \%$ & \\
\hline & Secondary & $73 \%$ & $27 \%$ & $\mathrm{X}^{2}=13.110, \mathrm{df}=2, \mathrm{p}=.001$ \\
\hline & Tertiary & $94 \%$ & $6 \%$ & \\
\hline \multirow[t]{3}{*}{ Lpg retailing experience } & $<5$ years & $89 \%$ & $11 \%$ & \\
\hline & $6-10$ years & $66 \%$ & $34 \%$ & $\mathrm{X}^{2}=23.685, \mathrm{df}=2, \mathrm{p}=<.001$ \\
\hline & $>10$ years & $67 \%$ & $33 \%$ & \\
\hline
\end{tabular}




\subsection{Cylinder Branding}

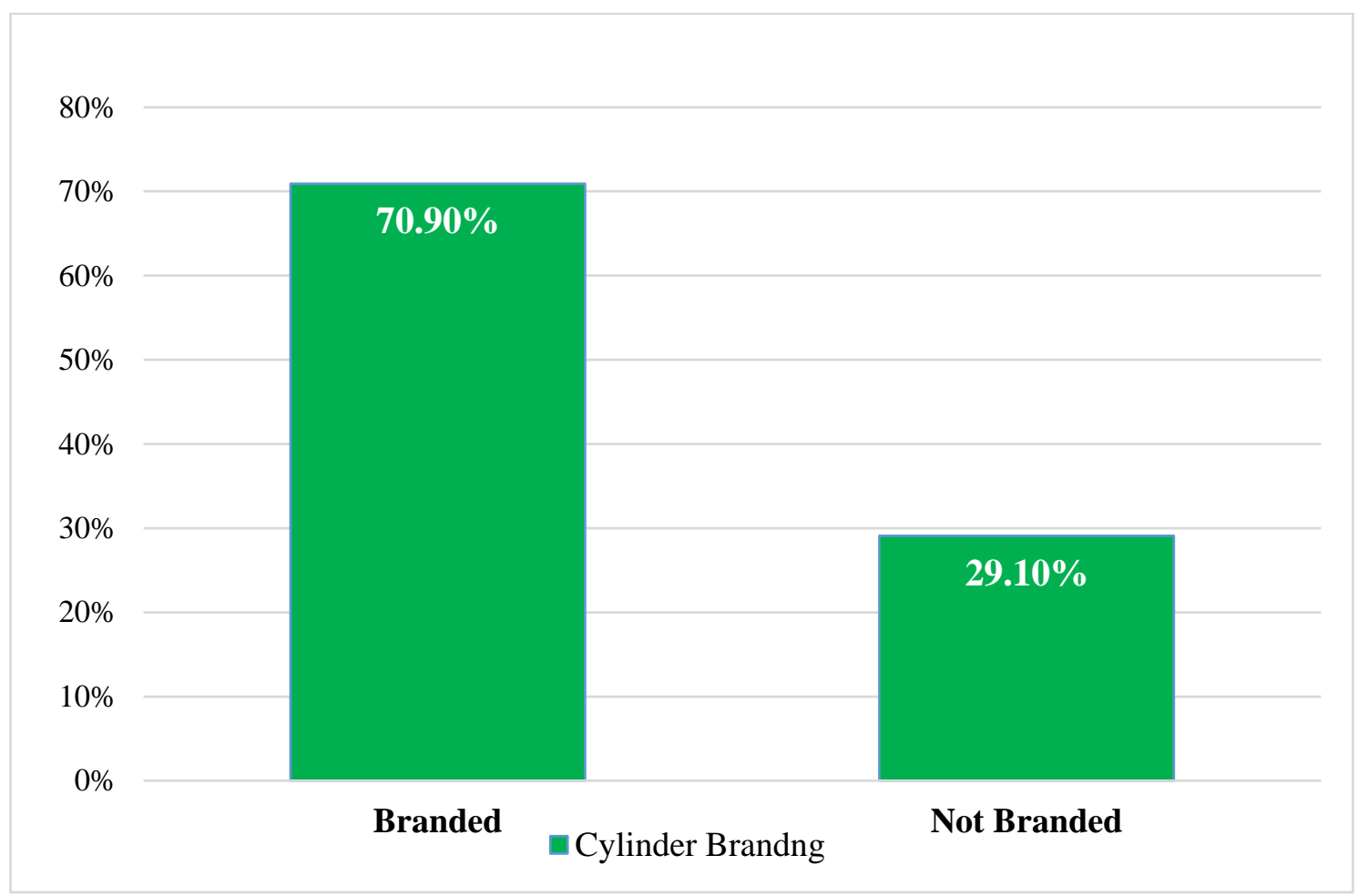

Figure 2: Cylinder branding

Unbranded Lpg cylinders may be a likely indication of illegal gas filling as no registered brand owner will circulate their gas in the market in an unbranded cylinder.

\subsection{EPRA Licensing}

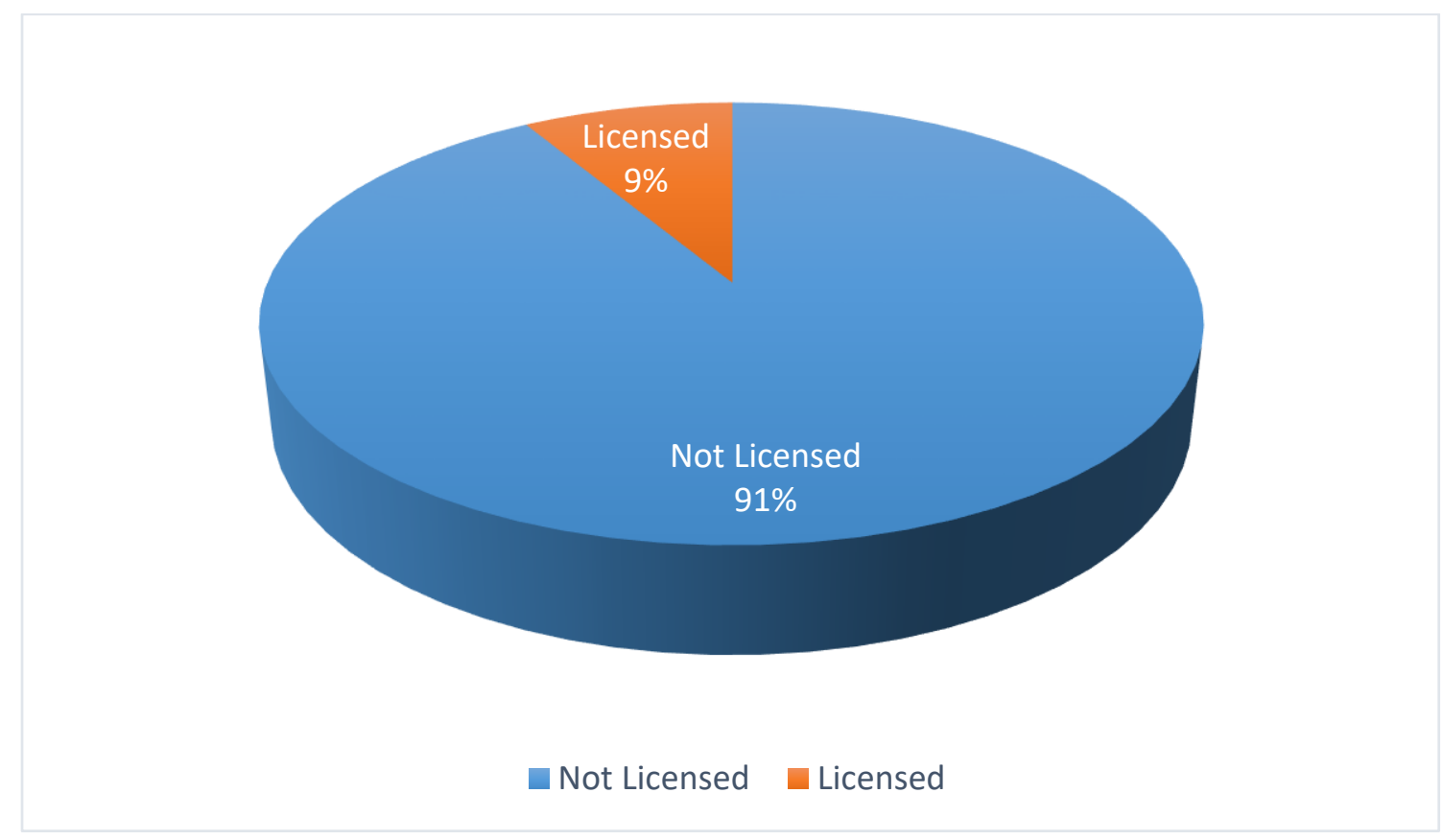

Figure 3: EPRA Licensing 


\subsection{Lpg cylinder filling and Non-destructive testing}

Table 5: 6Kg cylinder weight measurements and leak test.

\begin{tabular}{|c|c|c|c|c|c|c|c|c|}
\hline \multirow{2}{*}{ 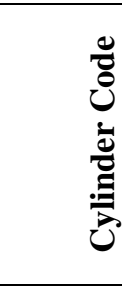 } & \multirow{2}{*}{ 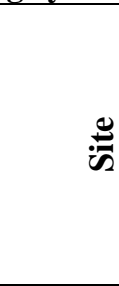 } & \multirow{2}{*}{ 象 } & \multirow{2}{*}{ 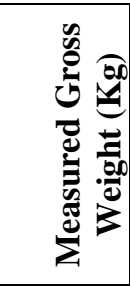 } & \multirow{2}{*}{ 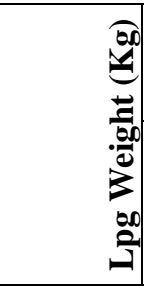 } & \multicolumn{2}{|c|}{ Leak test } & \multirow[b]{2}{*}{ Conforms } & \multirow[b]{2}{*}{ Reason } \\
\hline & & & & & Leak & No leak & & \\
\hline $6 \mathrm{C} 1$ & $\mathrm{~T} 1$ & 8.6 & 14.9 & 6.3 & No & Yes & No & $\mathrm{OF}$ \\
\hline $6 \mathrm{C} 2$ & $\mathrm{~T} 2$ & 7.8 & 14.0 & 6.2 & No & Yes & Yes & N/A \\
\hline $6 \mathrm{C} 3$ & T3 & 8.0 & 14.1 & 6.1 & No & Yes & Yes & N/A \\
\hline $6 \mathrm{C} 4$ & $\mathrm{~T} 4$ & 8.5 & 14.8 & 6.3 & No & Yes & No & $\mathrm{OF}$ \\
\hline $6 \mathrm{C} 5$ & T5 & 8.0 & 14.3 & 6.3 & No & Yes & No & $\mathrm{OF}$ \\
\hline $6 \mathrm{C} 6$ & T6 & 8.4 & 14.6 & 6.2 & No & Yes & Yes & $\mathrm{OF}$ \\
\hline $6 \mathrm{C} 7$ & $\mathrm{~T} 7$ & 8.3 & 14.7 & 6.4 & No & Yes & No & OF \\
\hline $6 \mathrm{C} 8$ & $\mathrm{~T} 8$ & 8.0 & 14.1 & 6.1 & Yes & No & No & $\mathrm{L}$ \\
\hline $6 \mathrm{C} 9$ & T9 & 8.3 & 14.1 & 5.8 & No & Yes & Yes & N/A \\
\hline $6 \mathrm{C} 10$ & $\mathrm{~T} 10$ & 8.2 & 14.0 & 5.8 & No & Yes & Yes & N/A \\
\hline $6 \mathrm{C} 11$ & T11 & 8.1 & 13.8 & 5.7 & No & Yes & No & UF \\
\hline $6 \mathrm{C} 12$ & $\mathrm{~T} 12$ & 8.5 & 14.6 & 6.1 & No & Yes & Yes & N/A \\
\hline $6 \mathrm{C} 13$ & $\mathrm{~T} 13$ & 8.6 & 14.6 & 6.0 & No & Yes & Yes & N/A \\
\hline $6 \mathrm{C} 14$ & T14 & 9.2 & 15.4 & 6.2 & No & Yes & Yes & N/A \\
\hline $6 \mathrm{C} 15$ & T15 & 8.5 & 14.4 & 5.9 & No & Yes & Yes & N/A \\
\hline $6 \mathrm{C} 16$ & $\mathrm{~J} 1$ & 8.5 & 14.1 & 5.6 & No & Yes & No & UF \\
\hline $6 \mathrm{C} 17$ & $\mathrm{~J} 2$ & 8.7 & 13.9 & 5.2 & Yes & No & No & L/UF \\
\hline $6 \mathrm{C} 18$ & $\mathrm{~J} 3$ & 8.5 & 13.9 & 5.4 & Yes & No & No & L/UF \\
\hline $6 \mathrm{C} 19$ & $\mathrm{~J} 4$ & 8.2 & 13.8 & 5.6 & No & Yes & No & UF \\
\hline $6 \mathrm{C} 20$ & J5 & 8.9 & 14.8 & 5.9 & No & Yes & Yes & N/A \\
\hline $6 \mathrm{C} 21$ & J6 & 8.0 & 14.2 & 6.2 & No & Yes & Yes & N/A \\
\hline $6 \mathrm{C} 22$ & J7 & 8.2 & 14.5 & 6.3 & No & Yes & No & $\mathrm{OF}$ \\
\hline $6 \mathrm{C} 23$ & $\mathrm{~J} 8$ & 8.7 & 14.8 & 6.1 & Yes & No & No & $\mathrm{L}$ \\
\hline $6 \mathrm{C} 24$ & J9 & 8.3 & 14.3 & 6.0 & No & Yes & Yes & N/A \\
\hline $6 \mathrm{C} 25$ & $\mathrm{~J} 10$ & 8.0 & 14.4 & 6.4 & No & Yes & No & $\mathrm{OF}$ \\
\hline $6 \mathrm{C} 26$ & J11 & 8.5 & 14.7 & 6.2 & No & Yes & Yes & N/A \\
\hline $6 \mathrm{C} 27$ & $\mathrm{~J} 12$ & 8.7 & 13.8 & 5.1 & Yes & No & No & $\mathrm{L}$ \\
\hline $6 \mathrm{C} 28$ & $\mathrm{~J} 13$ & 9.2 & 15.0 & 5.8 & No & Yes & Yes & N/A \\
\hline $6 \mathrm{C} 29$ & J14 & 8.4 & 14.6 & 6.2 & No & Yes & Yes & N/A \\
\hline $6 \mathrm{C} 30$ & $\mathrm{~J} 15$ & 8.3 & 14.2 & 5.9 & No & Yes & Yes & N/A \\
\hline \multicolumn{2}{|c|}{ Mean } & 8.4 & 14.4 & 6.0 & & & & \\
\hline \multicolumn{2}{|c|}{ SD } & 0.33415 & 0.3995 & $\mathbf{0 . 3 3 2 3 5}$ & & & & \\
\hline
\end{tabular}


Table 6: 13Kg cylinder weight measurements and leak test.

\begin{tabular}{|c|c|c|c|c|c|c|c|c|}
\hline \multirow{2}{*}{ 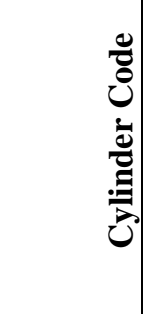 } & \multirow[b]{2}{*}{ 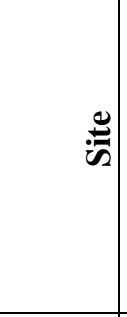 } & \multirow{2}{*}{ 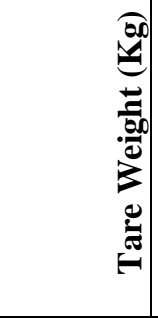 } & \multirow{2}{*}{ 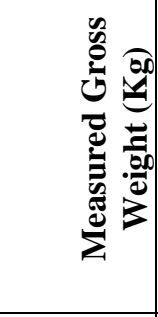 } & \multirow{2}{*}{ 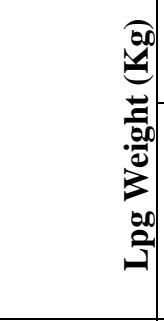 } & \multicolumn{2}{|c|}{ Leak test } & \multirow[b]{2}{*}{ Conforms } & \multirow[b]{2}{*}{ Reason } \\
\hline & & & & & Leak & No leak & & \\
\hline $13 \mathrm{C} 1$ & $\mathrm{~T} 1$ & 18.0 & 30.8 & 12.8 & No & Yes & Yes & N/A \\
\hline $13 \mathrm{C} 2$ & $\mathrm{~T} 2$ & 12.8 & 25.5 & 12.7 & No & Yes & No & UF \\
\hline $13 \mathrm{C} 3$ & T3 & 11.9 & 24.8 & 12.9 & No & Yes & Yes & N/A \\
\hline $13 \mathrm{C} 4$ & $\mathrm{~T} 4$ & 12.7 & 25.7 & 13.0 & No & Yes & Yes & N/A \\
\hline $13 \mathrm{C} 5$ & $\mathrm{~T} 5$ & 13.5 & 26.7 & 13.2 & No & Yes & Yes & N/A \\
\hline $13 \mathrm{C} 6$ & T6 & 13.0 & 26.2 & 13.2 & No & Yes & Yes & N/A \\
\hline $13 \mathrm{C} 7$ & $\mathrm{~T} 7$ & 11.9 & 25.1 & 13.2 & No & Yes & Yes & N/A \\
\hline $13 \mathrm{C} 8$ & $\mathrm{~T} 8$ & 12.0 & 25.4 & 13.4 & Yes & No & No & $\mathrm{L}$ \\
\hline $13 \mathrm{C} 9$ & T9 & 12.5 & 25.4 & 12.9 & No & Yes & Yes & N/A \\
\hline $13 \mathrm{C} 10$ & T10 & 12.9 & 26.0 & 13.1 & No & Yes & Yes & N/A \\
\hline $13 \mathrm{C} 11$ & T11 & 11.9 & 24.7 & 12.8 & No & Yes & Yes & N/A \\
\hline $13 \mathrm{C} 12$ & $\mathrm{~T} 12$ & 13.4 & 26.4 & 13.0 & No & Yes & Yes & N/A \\
\hline $13 \mathrm{C} 13$ & $\mathrm{~J} 1$ & 13.0 & 26.1 & 13.1 & No & Yes & Yes & N/A \\
\hline $13 \mathrm{C} 14$ & $\mathrm{~J} 2$ & 12.7 & 25.4 & 12.7 & No & Yes & No & UF \\
\hline $13 \mathrm{C} 15$ & $\mathrm{~J} 3$ & 13.9 & 26.7 & 12.8 & No & Yes & Yes & N/A \\
\hline $13 \mathrm{C} 16$ & $\mathrm{~J} 4$ & 14.0 & 26.6 & 12.6 & No & Yes & No & UF \\
\hline $13 \mathrm{C} 17$ & J5 & 12.7 & 26.0 & 13.3 & Yes & No & No & $\mathrm{L}$ \\
\hline $13 \mathrm{C} 18$ & J6 & 11.3 & 23.8 & 12.5 & No & Yes & Yes & N/A \\
\hline $13 \mathrm{C} 19$ & $\mathrm{~J} 7$ & 12.0 & 24.6 & 12.6 & No & Yes & No & UF \\
\hline $13 \mathrm{C} 20$ & J8 & 12.1 & 24.9 & 12.8 & No & Yes & Yes & N/A \\
\hline $13 \mathrm{C} 21$ & $\mathrm{~J} 9$ & 12.2 & 25.1 & 12.9 & No & Yes & Yes & N/A \\
\hline $13 \mathrm{C} 22$ & $\mathrm{~J} 10$ & 14.3 & 27.5 & 13.2 & No & Yes & Yes & N/A \\
\hline $13 \mathrm{C} 23$ & $\mathrm{~J} 11$ & 13.5 & 26.7 & 13.2 & No & Yes & Yes & N/A \\
\hline $13 \mathrm{C} 24$ & $\mathrm{~J} 12$ & 11.9 & 25.0 & 13.1 & No & Yes & Yes & N/A \\
\hline \multicolumn{2}{|c|}{ Mean } & 12.9 & 25.9 & 13.0 & & & & \\
\hline \multicolumn{2}{|c|}{ SD } & 1.30319 & 1.32539 & 0.23965 & & & & \\
\hline
\end{tabular}

\section{LEGEND}

N/A Not Applicable

UF Underfilled

L Leakage

OF Overfilled

Tolerance: $+-0.2 \mathrm{Kg}$. 


\subsection{Overall Lpg cylinder conformity Based on Measurements and Non-destructive tests}

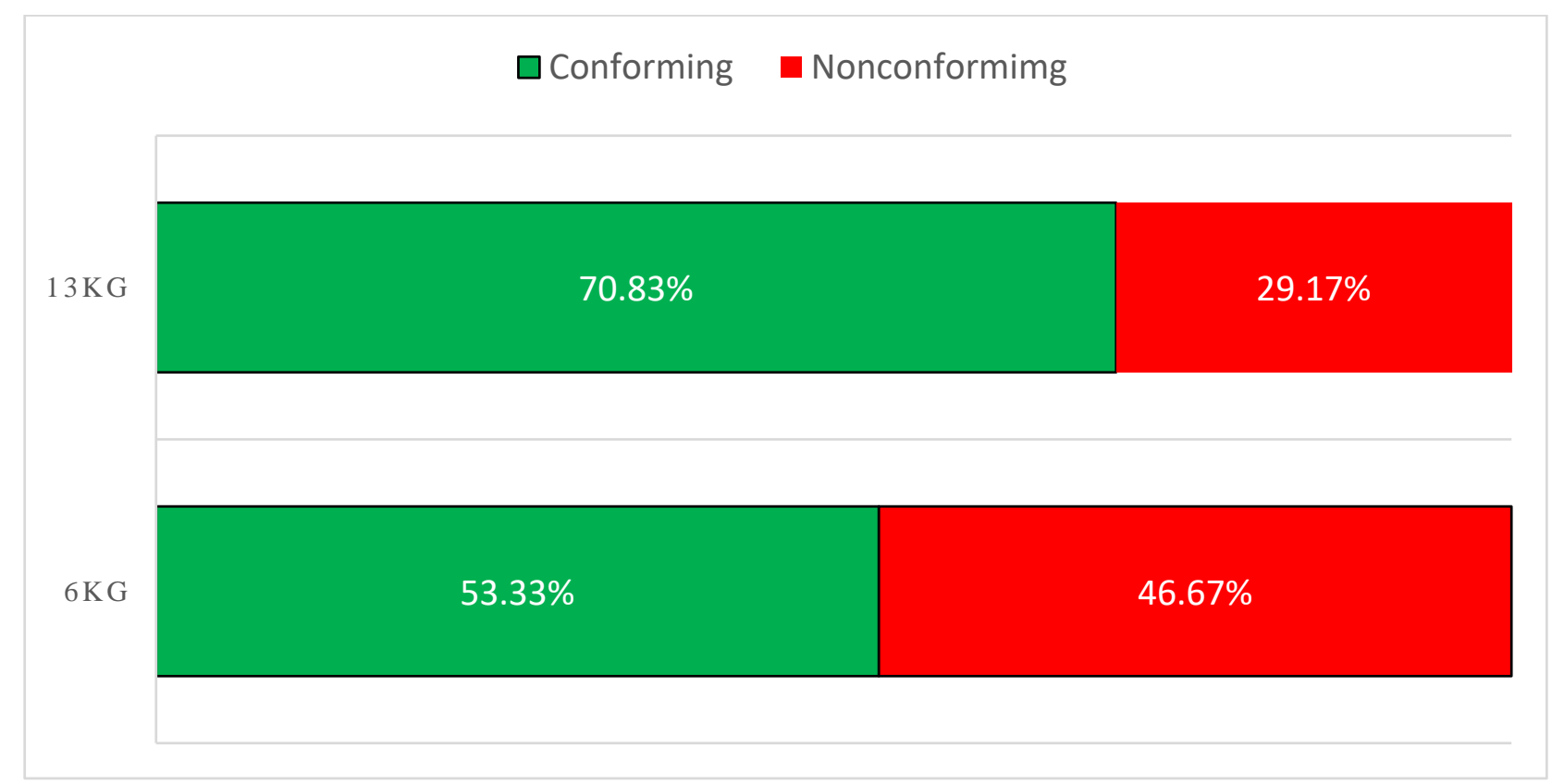

Figure 4: Overall Lpg cylinder conformity

\subsection{Challenges to good occupational safety and health practices}

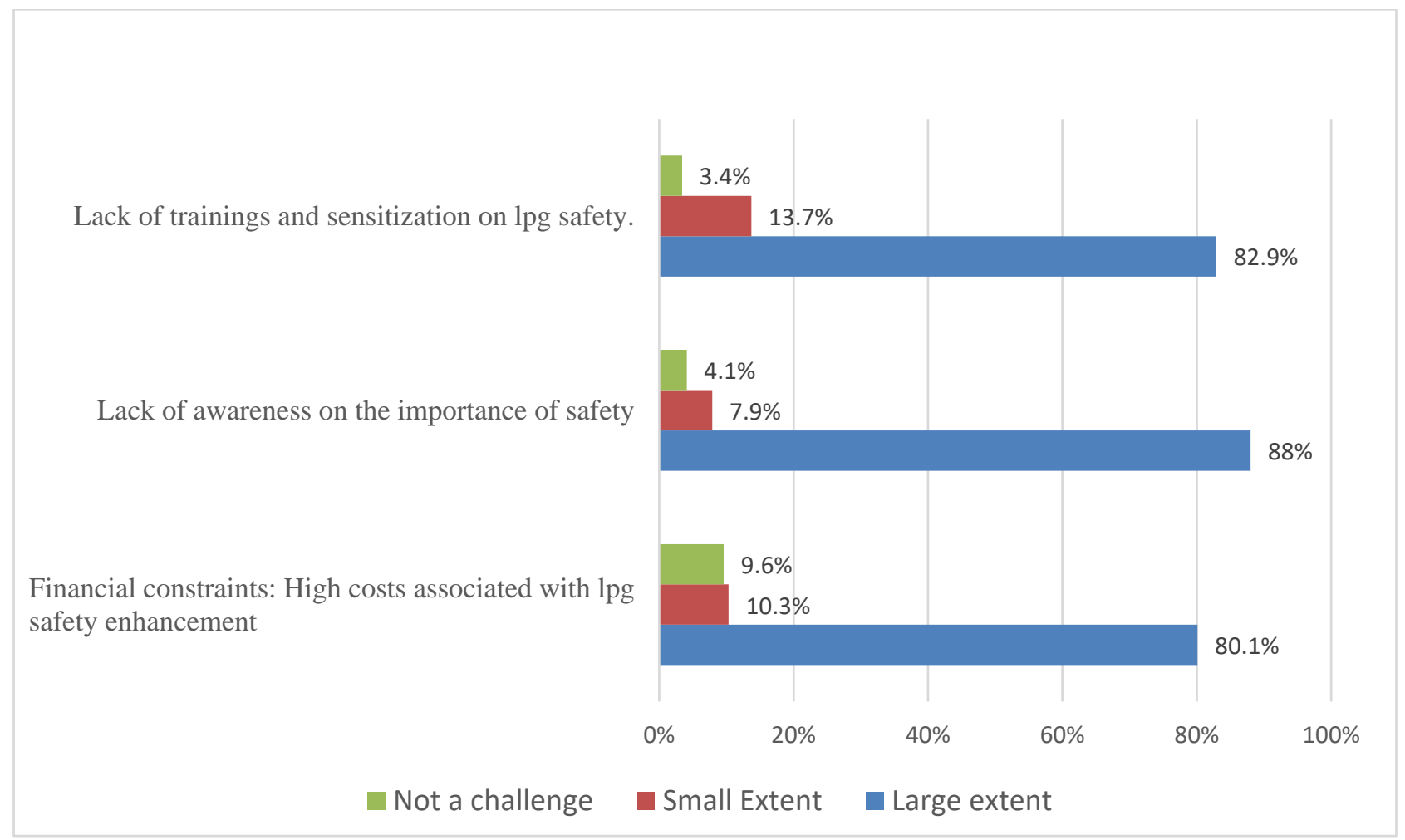

Figure 5: Challenges to good occupational safety and health practices 


\section{DISCUSSION AND CONCLUSION}

The study established that $71 \%$ of the Lpg cylinder retailers do not employ safe occupational safety and health practices in their Lpg retail operations-very worrying indeed-. At the same time, $16.7 \%$ of the $6 \mathrm{Kg}$ cylinders were found to be leaking from the valve. Leakages from the valve area maybe a likely indication of tampering with the valves during illegal gas refilling. $40 \%$ of the $6 \mathrm{Kg}$ cylinders were not conforming with regards to cylinder filling. Additionally, $8.3 \%$ of the $13 \mathrm{Kg}$ cylinders were as well found to be leaking from the valve. Similarly, $12.5 \%$ of the $13 \mathrm{Kg}$ cylinders were not conforming with respect to cylinder filling.

Overfilling of cylinders is unlikely to be done deliberately and a likely indication of faulty or poorly calibrated filling equipment. Underfilling of Lpg cylinders is most probably a deliberate act, which is disadvantageous to consumers as they don't get value for their money. In line with the consumer protection act of 2012, the customer should receive all the product purchased (World LPG Association, 2015)

The main challenges that hindered compliance with occupational safety and health requirements were ignorance, lack of awareness on the importance of safety in the lpg retail business, lack of pieces of training and sensitization on lpg safety, and the high costs associated with lpg safety enhancement.

The researcher recommends that the Energy and Petroleum Regulatory Authority (EPRA) should keep an up to date online register of licensed Lpg retailers, that is accessible to the public. The authority should also undertake routine inspections and compliance monitoring in all lpg retail centers. Lpg retailers should develop clear and simple safety policy guidelines, by consulting approved persons by the Directorate of Occupational Safety and Health Services (DOSHs), that will help ease the understanding of the safety requirements and consequently improve compliance. Besides, engineering breakthroughs should be sought after Lpg cylinder safety advancement.

\section{REFERENCES}

[1] Alli, B. (2008). Fundamental principles of occupational health and safety. Geneva: International Labour Organization.

[2] Babbie, E. (2002). Survey research methods. Belmont, CA: Wadsworth.

[3] Bartlett, J., Kotrlik, J., Higgins, C. (2001). Organizational research: determining appropriate sample size in survey research. Information Technology, Learning, and Perfomance Journal, Vol. 19, No. 1, Spring

[4] County Government of Kiambu. (2015). County Integrated Development Plan, 2013 - 2017. Kiambu: CGK.

[5] County Government of Kiambu. (2018). County Integrated Development Plan, 2018 - 2022. Kiambu: CGK.

[6] County Government of Kiambu. (2018). County Annual Development Plan 2018/2019. Kiambu: CGK.

[7] Government of Kenya. (2012). Consumer Protection Act No. 46 of 2012. Nairobi: Government Printer.

[8] Government of Kenya (2019). The Energy Act ( No 1. Of 2019).: Kenya Gazette Supplement No. 29 (Acts No.1). Nairobi : Government Printer

[9] Government of Kenya (2019). The Petroleum Act (No 2. Of 2019). The Petroleum (Liquefied Petroleum Gas ) Regulations, 2019: Legal Notice No. 100. Nairobi: Government Printer

[10] Kariithi, S, C.Mburu, \& R. Kinyua. (2017). Awareness of occupational safety and health issues in Roadway maintenance works in Nyeri County, Kenya International journal of innovative research and development, October 2017 Vol 6 Issue 10.

[11] Kothari, C. (2004). Research Methodology (Methods and Techniques). New Delhi: New Age International Publishers.

[12] Kothari, C.R \& Garg Gaurav. (2014). Research Methodology, Third Edition. New Delhi: New Age International Publishers.

[13] Occupational Safety and Health Act (2007). Nairobi: Government Press.

[14] Rubin, A. \& Babbie, R. (2012). Brooks/Cole empowerment series: Essential research methods for social work. Cengage Learning. ISBN:9781133709640.

[15] World Lpg Association. (2015). Guidelines for good safety practices in the LPG industry. WLPGA, International chamber of commerce

\section{AUTHORS}

First Author - James Karori Nyabuto, Student, Institute of Energy and Environmental Technology, Jomo Kenyatta University of Agriculture and Technology, Kenya., Jameskarori4@gmail.com.

Second Author - Dr. Charles Mburu, Senior Research Fellow, Institute of Energy and Environmental Technology, Jomo Kenyatta University of Agriculture and Technology, Kenya., mburu.charles@gmail.com.

Third Author - Dr. Margaret Gichuhi, Senior Research Fellow, Institute of Energy and Environmental Technology, Jomo Kenyatta University of Agriculture and Technology, Kenya., mgichuhi@jkuat.ac.ke.

Correspondence Author - James Karori Nyabuto, jameskarori4@gmail.com, +254728628110. 